\title{
INFLUÊNCIA DA TEMPERATURAE DA ÁGUA SOBRE A GERMINAÇÃO DE SEMENTES DE PARICÁ (Schizolobium amazonicum HUBER EX DUCKE - LEGUMINOSAE-CAESALPINIOIDEAE) ${ }^{1}$
}

\author{
MICHELEBRAULE PINTORAMOS², VANIAPALMEIRAVARELA ${ }^{3}$, MARIADEFÁTIMAFIGUEIREDO MELO 3
}

RESUMO - O Paricá (Schizolobium amazonicum Huber ex Ducke) é uma espécie florestal, pertencente à família Leguminosae-Caesalpinioideae, utilizada na recuperação de áreas degradadas e que apresenta destaque no mercado madeireiro. O presente trabalho foi conduzido com o objetivo de avaliar diferentes volumes de água no substrato e temperaturas para a germinação de sementes de $S$. amazonicum. As sementes foram imersas em ácido sulfúrico por 50 minutos, para superar a impermeabilidade do tegumento à água. Em seguida, foram semeadas em rolos de papel Germitest umedecidos com quantidades (mL.g-1 papel) de água equivalentes a 15; 2,0; 2,5 e 3,0 vezes a massa do substrato, sem adição posterior de água e mantidas em câmaras nas temperaturas constantes de 25,30 e $35^{\circ} \mathrm{C}$. Foram avaliados a porcentagem de germinação, o índice de velocidade de germinação e os comprimentos da raiz primária e do hipocótilo das plântulas normais. O delineamento experimental foi o inteiramente casualizado com os tratamentos em esquema fatorial $4 \times 3$. O melhor desempenho germinativo foi observado nas temperaturas de 25,30 e $35^{\circ} \mathrm{C}$, com as quantidades de água de 2,5 e 3,0 vezes a massa do papel. A velocidade do processo foi favorecida pelas temperaturas de 30 e $35^{\circ} \mathrm{C}$ e pela quantidade de água equivalente a 3,0 vezes a massa do papel. Ocorre maior desenvolvimento da raiz e do hipocótilo com a quantidade de água de 3,0 vezes a massa do papel a $25^{\circ} \mathrm{C}$.

Termos para indexação: espécie florestal, análise de sementes, volume de água.

\section{INFLUENCE OF TEMPERATUREAND WATER ON PARICÁ (Schizolobium amazonicum HUBER EXDUCKE-LEGUMINOSAE-CAESALPINIOIDEAE) SEED GERMINATION}

\begin{abstract}
Paricá (Schizolobium amazonicum Huber ex Ducke) is a tree species of the Leguminosae-Caesalpinioideae botanical family. This species has been used in rehabilitation programs for of degraded areas and it has good acceptance at local timber markets as well. This study aimed to evaluate the effects of different water contents in the substrate and temperatures on germination of S. amazonicum. Seeds were immersed in sulfuric acid for 50 minutes in order to break the impermeability of the seed-coat. After this, they were seeded over Germitest paper rolls wetted with water contents ( $\mathrm{mL} . \mathrm{g}^{-1}$ paper) equivalent to $1.5,2,2.5$ and 3 times the substrate mass without new water addition, and they were maintained in chambers at constant temperatures of 25,30 and $35^{\circ} \mathrm{C}$. In addition to the germination percentage, the speed of germination index and length of primary roots and hypocotyl of normal seedlings were also evaluated. A completely randomized design was used with a $4 \times 3$ factorial. The best germination performance was observed at temperatures of 25,30 and $35^{\circ} \mathrm{C}$ combining with water contents of 2.5 and 3 times the paper mass. The germination speed was favored by temperatures of 30 and $35^{\circ} \mathrm{C}$ as well as by the largest water content, which corresponded to 3 times the paper mass. The best results related to root and hypocotyl development were observed in treatments involving water content 3 times the paper mass at a temperature of $25^{\circ} \mathrm{C}$.
\end{abstract}

Index terms: forest species, seeds analysis, water volume.

\footnotetext{
${ }^{1}$ Submetido em 25/10/2004. Aceito para publicação em 04/11/2005, pesquisa financiada pela FAPEAM (www.fapeam.am.gov.br);

${ }^{2}$ M.Sc., Instituto Nacional de Pesquisas da Amazônia (INPA), Cx. Postal
}

478, CEP 69.060-001 - Manaus-AM, mbraule@inpa.gov.br;

${ }^{3}$ M.Sc., Pesquisadoras, INPA, vaniav@inpa.gov.br, ffmelo@inpa.gov.br. 


\section{INTRODUÇÃO}

A espécie Schizolobium amazonicum Huber ex Ducke (Paricá), pertencente à família Leguminosae-Caesalpinioideae, é uma árvore de grande porte e rápido crescimento (Ducke, 1949; Rondon, 2000). Ocorre em mata primária e secundária de terra-firme e várzea alta e está distribuída nos estados do Amazonas e Pará (Ducke, 1949; Rizzini, 1971). A madeira da espécie apresenta elevado interesse econômico e tem como principais aplicações caixas, forros, pranchetas, palitos, canoas, aeromodelos, brinquedos e papel (Rizzini, 1971).

Nos testes de germinação realizados em laboratório, fatores como dormência, quantidade de água, luz, temperatura, oxigênio e ocorrência de agentes patogênicos, influenciam significativamente nas respostas germinativas (Ramos e Bianchetti, 1984; Popinigis, 1985; Brasil, 1992; Carvalho e Nakagawa, 2000). Dessa forma, o conhecimento das condições adequadas para a germinação de cada espécie tornase imprescindível quando o objetivo for minimizar a discrepância entre os resultados de diferentes trabalhos.

A temperatura é um fator de grande influência sobre as reações bioquímicas que regulam o metabolismo necessário para iniciar o processo de germinação, e em conseqüência sobre a porcentagem e velocidade do processo (Carvalho e Nakagawa, 2000). A temperatura ótima é aquela na qual a semente expressa seu potencial máximo de germinação e as temperaturas máxima e mínima, são os pontos críticos onde acima e abaixo dos quais, respectivamente, não ocorre germinação (Popinigis, 1985; Mayer e Poljakoff-Mayber, 1989). As espécies, entretanto, apresentam comportamento variável quanto à temperatura de germinação, não havendo uma única temperatura ótima para todas as plantas.

A faixa de 20 a $30^{\circ} \mathrm{C}$ foi considerada, por Borges e Rena (1993), como a mais adequada para a germinação de grande número de espécies florestais subtropicais e tropicais. Ferraz e Varela (2003), trabalhando com trinta espécies florestais da Amazônia, verificaram que em temperaturas entre 25 e $35^{\circ} \mathrm{C}$ o processo de germinação foi mais eficiente para a maioria das espécies estudadas. Andrade e Pereira (1994) indicaram para Cedrela odorata as temperaturas de 25 e $30^{\circ} \mathrm{C}$ como as que proporcionaram maior porcentagem de germinação. Miranda e Ferraz (1999) e Varela et al. (1999) observaram que as sementes das espécies florestais amazônicas Maquira sclerophylla e Ceiba pentandra apresentaram melhor desempenho germinativo na temperatura de $30^{\circ} \mathrm{C}$.

A umidade do substrato onde é realizada a semeadura constitui um dos fatores essenciais para desencadear o processo de germinação. Durante esse processo, a absorção de água tem como principais funções promover o amolecimento do tegumento da semente, o aumento do embrião e dos tecidos de reserva, favorecendo a ruptura do tegumento, a difusão gasosa e a emergência da raiz primária. A água é importante, ainda, para a diluição do protoplasma, permitindo a difusão de hormônios e conseqüentemente a ativação de sistemas enzimáticos; com isso, desenvolvem-se a digestão, a translocação e a assimilação das reservas resultando no crescimento do embrião (Marcos Filho, 1986).

O substrato deve permanecer uniformemente úmido durante os testes de germinação realizados em laboratório, visando suprir as sementes da quantidade de água necessária para sua germinação e desenvolvimento. Por outro lado, o excesso de umidade provoca um decréscimo na germinação, pois dificulta a respiração e reduz todo o processo metabólico resultante, além de aumentar a incidência de fungos, levando à redução na viabilidade (Figliolia et al., 1993).

Os estudos de germinação relacionados ao nível de umidade do substrato são pioneiros na Tecnologia de Sementes de espécies florestais da Amazônia, e podem contribuir para aprimorar a condução dos testes de germinação, reduzindo a discrepância entre os resultados obtidos em diferentes laboratórios. Pesquisas sobre as exigências de água em sementes de espécies agrícolas realizadas por Phaneendranath (1980), Tanaka et al. (1991) e Novembre e Marcos Filho (1999) têm mostrado resultados consistentes, quando a água é controlada através de cálculo baseado na relação volume de água e peso do substrato do papel, sem hidratação posterior.

Para a espécie $S$. amazonicum, estão disponíveis na literatura somente estudos relacionados às técnicas de plantio (Rondon, 2000, 2002); entretanto, não existem pesquisas abordando a influência da quantidade de água no substrato e da temperatura sobre a germinação das sementes. Diante da carência de informações sobre as sementes desta espécie, o presente trabalho foi conduzido para avaliar a germinação de Schizolobium amazonicum em função de diferentes volumes de água e temperaturas.

\section{MATERIAL E MÉTODOS}

As sementes de $S$. amazonicum utilizadas neste estudo foram fornecidas pela Associação das Indústrias Exportadoras de Madeiras do Estado do Pará (AIMEX) e coletadas em Rondônia em outubro/2003.

Inicialmente, as sementes foram tratadas para a superação da impermeabilidade do tegumento à água, utilizando-se ácido 
sulfúrico (96\%) por 50 minutos. Em seguida, foram semeadas em rolos de papel Germitest, umedecidos com quantidades de água equivalentes a 1,$5 ; 2,0 ; 2,5$ e 3,0 vezes a massa do substrato, sem adição posterior de água, com três folhas por rolo. Os rolos foram acondicionados em sacos de plástico de $0,04 \mathrm{~mm}$ de espessura, com a finalidade de evitar a perda de água por evaporação. Para cada tratamento, utilizaram-se quatro repetições de 25 sementes, mantidas nas temperaturas constantes de 25,30 e $35^{\circ} \mathrm{C}$. Os testes de germinação foram conduzidos, com fotoperíodo de 12 horas, em câmaras providas de lâmpadas fluorescentes de luz branca fria e fluxo luminoso de $10 \mathrm{mmol} \cdot \mathrm{m}^{-2} \cdot \mathrm{s}^{-1}$ de radiação PAR (radiação fotossinteticamente ativa).

As avaliações foram efetuadas diariamente após a instalação do teste, por um período de 15 dias, quando o experimento foi encerrado. Considerou-se como semente germinada aquela que apresentou todas as estruturas essenciais desenvolvidas (Brasil, 1992). Foram calculados a porcentagem de plântulas normais e o índice de velocidade de germinação, conforme equação proposta por Maguire (1962):

Foram medidos os comprimentos da raiz primária e do hipocótilo, com o auxílio de régua milimetrada, tomando-se aleatoriamente dez plântulas por repetição de cada tratamento. Os resultados médios foram expressos em milímetros.

O delineamento experimental foi o inteiramente casualizado, em esquema fatorial $4 \times 3$ (quantidades de água no substrato $\mathrm{x}$ temperatura), com quatro repetições de 25 sementes para cada tratamento. Utilizou-se a transformação em $\operatorname{arcsen} \sqrt{X / 100}$, para os dados em porcentagens, e em $\sqrt{(\mathrm{x}+0,05)}$ para os dados de comprimento da raiz primária e do hipocótilo, com o objetivo de normalizar a distribuição. Os resultados foram submetidos à análise de variância e as médias comparadas pelo teste de Tukey $(5 \%)$.

\section{RESULTADOS E DISCUSSÃO}

Os resultados de porcentagem de germinação das sementes de S. amazonicum obtidas em diferentes temperaturas e quantidades de água no substrato apresentaram diferenças significativas para a interação entre os fatores estudados (Tabela 1). Nas temperaturas de 25 e $35^{\circ} \mathrm{C}$, foram observados valores estatisticamente superiores para as quantidades de água de 2,0; 2,5 e 3,0 vezes a massa do substrato, quando comparados com 1,5 vezes. Entretanto, os valores observados para a quantidade de água de 2,0 vezes a massa do papel são numericamente inferiores aos apresentados para 2,5 e 3,0 vezes, para ambas as temperaturas. Esse comportamento para as quantidades de água de 1,5 e 2,0 vezes a massa do substrato pode indicar que a redução de água no substrato é desfavorável para processo germinativo dessa espécie. A temperatura de $30^{\circ} \mathrm{C}$ apresentou melhores resultados para as quantidades de água de 2,5 e 3,0 vezes a massa do substrato, com $85 \%$ de germinação em ambos os tratamentos. Para todas as temperaturas utilizadas, a quantidade de água equivalente a 1,5 vezes a massa do papel foi prejudicial, apresentando valores entre 7 e $40 \%$ de germinação.

Existem poucos estudos disponíveis na literatura sobre a influência do umedecimento do substrato na germinação de sementes de espécies florestais, sendo a maioria referente a espécies agronômicas. Mesquita (2004), em recente trabalho com a espécie florestal nativa da Amazônia Angelim-pedra (Dinizia excelsa), verificou que as temperaturas e quantidades de água no substrato não influenciaram a germinação da espécie. Os resultados observados para a espécie $S$. amazonicum, comparados com $D$. excelsa, demonstraram que a influência da temperatura e das quantidades de água no substrato podem ser bastante diferentes entre as espécies florestais amazônicas.

Trabalhando com sementes de maxixe (Cucumis anguria) Gentil e Torres (2001) observaram que as quantidades de água de 1,$0 ; 1,5 ; 2,0$ e 2,5 vezes a massa do papel a $25^{\circ} \mathrm{C}$ foram mais favoráveis para a germinação. Para as sementes de amendoim houve variação de acordo com o lote estudado, sendo os melhores resultados obtidos com 2,0 vezes a massa do papel (Tanaka et al., 1991). Segundo Vicente et al. (1969),

TABELA 1. Germinação (\%) de sementes de $S$. amazonicum obtidas sob três temperaturas e quantidades de água no substrato

\begin{tabular}{ccccc}
\hline Quantidade de água & \multicolumn{3}{c}{ Temperatura $\left({ }^{\circ} \mathrm{C}\right)$} & \multirow{2}{*}{ Médias } \\
\cline { 2 - 4 }$\left(\mathrm{mL} \cdot \mathrm{g}^{-1}\right.$ de papel $)$ & 25 & 30 & 35 & \\
\hline 1,5 & $40 \mathrm{Ba}$ & $34 \mathrm{Ca}$ & $7 \mathrm{Bb}$ & $27 \mathrm{C}$ \\
2,0 & $66 \mathrm{Aa}$ & $63 \mathrm{Ba}$ & $72 \mathrm{Aa}$ & $67 \mathrm{~B}$ \\
2,5 & $82 \mathrm{Aa}$ & $85 \mathrm{Aa}$ & $84 \mathrm{Aa}$ & $84 \mathrm{~A}$ \\
3,0 & $74 \mathrm{Aa}$ & $85 \mathrm{Aa}$ & $86 \mathrm{Aa}$ & $82 \mathrm{~A}$ \\
\hline Médias & 65,5 & 66,8 & 62,3 & \\
\hline $\mathrm{F}_{\mathrm{t}}=2,88 \mathrm{~ns}$ & $\mathrm{CV}=10,78 \%$ & & & \\
$\mathrm{~F}_{\mathrm{q}}=109,28^{* *}$ & & & & \\
$\mathrm{~F}_{\mathrm{txq}}=9,27^{* *}$ & & & & \\
\hline
\end{tabular}

Médias seguidas pela mesma letra maiúscula na coluna e minúscula na linha não diferem entre si, pelo teste de Tukey (5\%).

ns - não significativo para temperatura; ** - significativo a $1 \%$ de probabilidade para quantidade de água e interação. 
a germinação mais favorável pode não ser induzida por somente um volume de água, mas por diversos volumes que ocupam uma faixa de amplitude específica, conforme a espécie.

A interação entre os fatores estudados indicou que a germinação das sementes de $S$. amazonicum submetidas às quantidades de água de 2,0, 2,5 e 3,0 vezes a massa do substrato não foi influenciada pelas temperaturas testadas (Tabela 1). Resultado semelhante foi observado por Mesquita (2004), onde as temperaturas não influenciaram a germinação de $D$. excelsa para as quantidades de água de 1,5, 2,0, 2,5 e 3,0 vezes a massa do papel.

A quantidade de água de 1,5 vezes a massa do papel foi prejudicial para a germinação independente das temperaturas, especialmente para a de $35^{\circ} \mathrm{C}$. Esse comportamento pode estar relacionado com o aumento da temperatura $\left(35^{\circ} \mathrm{C}\right)$ que acentuou o processo de evaporação da água contida no substrato, reduzindo a $7 \%$ a porcentagem final de germinação.

Os dados observados para $S$. amazonicum estão de acordo com os propostos por Borges e Rena (1993) de que as sementes de grande número de espécies florestais subtropicais e tropicais mostram potencial máximo de germinação na faixa de temperatura entre 20 e $30^{\circ} \mathrm{C}$. Espécies nativas da floresta amazônica também apresentaram melhores taxas de germinação nas temperaturas de $25^{\circ} \mathrm{C}$ (Triplaris surinamensis), e de 25 e $30^{\circ} \mathrm{C}$ (Dipteryx alata) (Carneiro et al., 1997). Para as sementes de Leandra breviflora, Tibouchina benthamiana e T. moricandiana, espécies da Mata Atlântica, as temperaturas de 20,25 e $30^{\circ} \mathrm{C}$ foram as melhores para emissão da raiz primária (Andrade, 1995).

A velocidade do processo germinativo, representado pelo índice de velocidade de germinação (IVG), foi influenciada pela temperatura, quantidade de água no substrato e interação entre esses fatores (Tabela 2).

Na temperatura de $25^{\circ} \mathrm{C}$, os melhores resultados de IVG foram obtidos para as quantidades de água de 2,0,2,5 e 3,0 vezes a massa do papel, com valores de 3,0, 4,2 e 4,3. Para a temperatura de $30^{\circ} \mathrm{C}$, os tratamentos com 2,5 e 3,0 vezes a massa do substrato resultaram em maiores índices de velocidade, 5,3 e 5,8, respectivamente. A maior velocidade de germinação na temperatura de $35^{\circ} \mathrm{C}$ foi obtida com a quantidade de água de 3,0 vezes a massa do papel quando comparada aos demais tratamentos, resultando no valor de 5,9. De forma semelhante ao observado na porcentagem de germinação, a quantidade de água de 1,5 vezes a massa do papel mostrou-se prejudicial para a velocidade de germinação em todas as temperaturas testadas. Isso se deve à baixa disponibilidade de água nesse tratamento que, provavelmente, tornou-se insuficiente para desencadear os processos metabólicos da germinação dessa espécie.

Para as quantidades de água equivalentes a 1,5, 2,0 e 2,5 vezes a massa do papel, as temperaturas testadas não influenciaram significativamente o índice de velocidade de germinação. $\mathrm{O}$ tratamento com 3,0 vezes a massa do substrato apresentou melhores resultados para o IVG nas temperaturas de 30 e $35^{\circ} \mathrm{C}$, com valores de 5,8 e 5,9 .

Os resultados de índice de velocidade de germinação obtidos foram melhores quando utilizou-se maiores quantidades de água (Tabela 2). Bisognin et al. (1991), para as sementes de Porongo, indicaram que a menor velocidade foi observada com quantidade de água equivalente a 3,0 vezes a massa do substrato. Para a espécie $D$. excelsa, a velocidade do processo não foi influenciada pelas quantidades de água no substrato e pelas temperaturas de germinação (Mesquita, 2004).

As médias de comprimento da raiz primária das sementes foram influenciadas pelas temperaturas, quantidades de água no substrato e interação entre esses fatores (Tabela 3 ).

Nas temperaturas de 25 e $30^{\circ} \mathrm{C}$ foram obtidos maiores comprimentos de raiz primária para a quantidade de água de 3,0 vezes a massa do papel, com 10,4 e $6,9 \mathrm{~cm}$ de comprimento, respectivamente. Na temperatura de $35^{\circ} \mathrm{C}$, melhor resultado foi obtido com a quantidade de água de 2,5 vezes, com 5,2cm de comprimento da raiz.

O desenvolvimento da raiz primária para as quantidades de água de 1,$5 ; 2,0$ e 2,5 vezes a massa do papel não foi influenciado pelas temperaturas testadas. Para 3,0 vezes a massa do papel, a temperatura de $25^{\circ} \mathrm{C}$ foi mais favorável para o desenvolvimento da raiz, apresentando $10,4 \mathrm{~cm}$ de

TABELA 2. Índice de velocidade de germinação (IVG) das sementes de $S$. amazonicum em três temperaturas e quatro quantidades de água no substrato

\begin{tabular}{|c|c|c|c|c|}
\hline \multirow{2}{*}{$\begin{array}{l}\text { Quantidade de água } \\
\text { (mL.g }{ }^{-1} \text { de papel) }\end{array}$} & \multicolumn{3}{|c|}{ Temperatura $\left({ }^{\circ} \mathrm{C}\right)$} & \multirow{2}{*}{ Médias } \\
\hline & 25 & 30 & 35 & \\
\hline 1,5 & $1,5 \mathrm{Ba}$ & $1,4 \mathrm{Ca}$ & $0,4 \mathrm{Ca}$ & $1,1 \mathrm{C}$ \\
\hline 2,0 & $3,0 \mathrm{Aa}$ & $3,6 \mathrm{Ba}$ & $3,8 \mathrm{Ba}$ & $3,5 \mathrm{~B}$ \\
\hline 2,5 & $4,2 \mathrm{Aa}$ & $5,3 \mathrm{Aa}$ & $5,1 \mathrm{ABa}$ & $4,9 \mathrm{~A}$ \\
\hline 3,0 & $4,3 \mathrm{Ab}$ & $5,8 \mathrm{Aa}$ & $5,9 \mathrm{Aa}$ & $5,3 \mathrm{~A}$ \\
\hline Médias & $3,3 \mathrm{~b}$ & $4,0 \mathrm{a}$ & $3,8 \mathrm{ab}$ & \\
\hline $\begin{array}{l}\mathrm{F}_{\mathrm{t}}=4,66 * \\
\mathrm{~F}_{\mathrm{q}}=84,69 * * \\
\mathrm{~F}_{\mathrm{txq}}=2,72 *\end{array}$ & \multicolumn{4}{|c|}{$\mathrm{CV}=19,56 \%$} \\
\hline
\end{tabular}

Médias seguidas pela mesma letra maiúscula na coluna e minúscula na linha não diferem entre si, pelo teste de Tukey (5\%).

* ,** - significativo para temperatura, quantidade de água e interação. 
TABELA 3. Comprimento da raiz primária $(\mathrm{mm})$ de sementes de S. amazonicum em três temperaturas e quatro quantidades de água no substrato

\begin{tabular}{|c|c|c|c|c|}
\hline \multirow{2}{*}{$\begin{array}{l}\text { Quantidade de água } \\
\text { (mL.g }{ }^{-1} \text { de papel) }\end{array}$} & \multicolumn{3}{|c|}{ Temperatura $\left({ }^{\circ} \mathrm{C}\right)$} & \multirow{2}{*}{ Médias } \\
\hline & 25 & 30 & 35 & \\
\hline 1,5 & $3,0 \mathrm{Ca}$ & $3,5 \mathrm{Ba}$ & $2,8 \mathrm{Ba}$ & $3,1 \mathrm{C}$ \\
\hline 2,0 & $4,4 \mathrm{BCa}$ & $3,7 \mathrm{Ba}$ & $4,7 \mathrm{ABa}$ & $4,3 \mathrm{BC}$ \\
\hline 2,5 & 6,4 Ba & $5,4 \mathrm{ABa}$ & 5,2 Aa & $5,7 \mathrm{AB}$ \\
\hline 3,0 & $10,4 \mathrm{Aa}$ & $6,9 \mathrm{Ab}$ & $4,5 \mathrm{ABc}$ & $7,3 \mathrm{~A}$ \\
\hline Médias & $6,1 \mathrm{a}$ & $4,9 \mathrm{ab}$ & $4,3 \mathrm{~b}$ & \\
\hline $\begin{array}{l}\mathrm{F}_{\mathrm{t}}=5,40^{* *} \\
\mathrm{~F}_{\mathrm{q}}=20,78^{* *} \\
\mathrm{~F}_{\mathrm{txq}}=3,81^{* *}\end{array}$ & \multicolumn{2}{|c|}{$\mathrm{CV}=19,12 \%$} & & \\
\hline
\end{tabular}

Médias seguidas pela mesma letra maiúscula na coluna e minúscula na linha não diferem entre si, pelo teste de Tukey (5\%).

** - significativo para temperatura, quantidade de água e interação.

comprimento. Para a espécie $D$. excelsa, foram observados resultados semelhantes quanto ao comprimento da raiz primária, pois as temperaturas de 25,30 e $35^{\circ} \mathrm{C}$ não influenciaram o desenvolvimento da raiz (Mesquita, 2004). Por outro lado, a mesma autora cita que as quantidades de água que proporcionaram os maiores comprimentos de raiz primária foram 1,5 e 2,0 vezes a massa do papel a $25^{\circ} \mathrm{C}, 1,5$ vezes a $30^{\circ} \mathrm{C}$ e 3,0 vezes a $35^{\circ} \mathrm{C}$, diferindo dos resultados encontrados para $S$. amazonicum.

O comprimento do hipocótilo observado na germinação de sementes foi influenciado apenas pelo fator quantidade de água (Tabela 4), onde o valor equivalente a 3,0 vezes a massa do papel proporcionou seu maior desenvolvimento. Enquanto que Mesquita (2004) constatou influência da temperatura no

TABELA 4. Comprimento do hipocótilo $(\mathrm{mm})$ de sementes de $S$. amazonicum em três temperaturas e quatro quantidades de água no substrato

\begin{tabular}{ccccc}
\hline $\begin{array}{c}\text { Quantidade de água } \\
\left(\mathrm{mL} \cdot \mathrm{g}^{-1} \text { de papel }\right)\end{array}$ & \multicolumn{3}{c}{ Temperatura $\left({ }^{\circ} \mathrm{C}\right)$} & Médias \\
\cline { 2 - 4 } & 25 & 30 & 35 & \\
\hline 1,5 & 2,3 & 2,7 & 2,8 & $2,6 \mathrm{D}$ \\
2,0 & 3,9 & 3,8 & 4,1 & $3,9 \mathrm{C}$ \\
2,5 & 4,5 & 5,6 & 5,2 & $5,1 \mathrm{~B}$ \\
3,0 & 6,9 & 6,7 & 6,7 & $6,6 \mathrm{~A}$ \\
\hline Médias & 4,4 & 4,6 & 4,7 & \\
\hline $\mathrm{F}_{\mathrm{t}}=0,62 \mathrm{~ns}$ & $\mathrm{CV}=14,39 \%$ & & \\
$\mathrm{~F}_{\mathrm{q}}=48,67 * *$ & & & \\
$\mathrm{~F}_{\mathrm{txq}}=0,65 \mathrm{~ns}$ & & & \\
\hline
\end{tabular}

Médias seguidas pela mesma letra maiúscula na coluna não diferem entre si, pelo teste de Tukey (1\%).

ns - não significativo para temperatura; ** - significativo para quantidade de água. desenvolvimento do hipocótilo da espécie Angelim pedra, sendo que a 30 e $35^{\circ} \mathrm{C}$ ocorreram os melhores resultados, também verificaram que quantidades de água acima de 2,0 vezes a massa do papel proporcionaram maiores comprimentos do hipocótilo.

A menor quantidade de água testada (1,5 vezes a massa do papel) foi insuficiente para o desenvolvimento tanto da raiz quanto do hipocótilo. Esses resultados confirmam os observados para a porcentagem de germinação e índice de velocidade de germinação.

Considerando as quatro variáveis estudadas, as temperaturas de 30 e $35^{\circ} \mathrm{C}$ com a quantidade de água de 3,0 vezes a massa do papel resultaram em maiores porcentagens de germinação e maiores índices de velocidades do processo de germinação. Por outro lado, para o desenvolvimento da raiz e do hipocótilo da espécie, os melhores resultados foram obtidos na temperatura de $25^{\circ} \mathrm{C}$ com a quantidade de água de 3,0 vezes a massa do papel. Os resultados obtidos nesse estudo demonstraram que $S$. amazonicum necessita de maior quantidade de água para acelerar o processo germinativo e ao mesmo tempo favorecer o desenvolvimento das plântulas.

\section{CONCLUSÕES}

O melhor desempenho germinativo foi observado nas temperaturas de 25,30 e $35^{\circ} \mathrm{C}$, com as quantidades de água de 2,5 e 3,0 vezes a massa do papel.

A velocidade do processo foi favorecida pelas temperaturas de 30 e $35^{\circ} \mathrm{C}$ e pela quantidade de água equivalente a 3,0 vezes a massa do papel.

Ocorre maior desenvolvimento da raiz e do hipocótilo com a quantidade de água de 3,0 vezes a massa do papel a $25^{\circ} \mathrm{C}$.

\section{REFERÊNCIAS}

ANDRADE, A.C.S. Efeito da luz e temperatura na germinação de Leandra breviflora Cogn., Tibouchina benthamiana Cogn., Tibouchina glandiflora Cogn., e Tibouchina moricandiana (DC.)Boill (Melastomataceae). Revista Brasileira de Sementes, Brasília, v. 20, n.2, p.29-35, 1995.

ANDRADE, A.C.S.; PEREIRA, T.S. Efeito do substrato e da temperatura na germinação e no vigor de sementes de cedro Cedrela odorata L. (Meliaceae). Revista Brasileira de Sementes, Brasília, v.16, n.1, p.34-40, 1994.

BISOGNIN, D.A.; IRIGON, D.L.; MARTINAZZO, A.A. Teste de germinação em porongo - Lagenaria siceraria (Mol.) Standi. Ciência Rural, Santa Maria, v.21, n.2, p.159-167, 1991.

BORGES, E.E.I.; RENA, A.B. Germinação de sementes. In: 
AGUIAR, I.B. ; PINÃ-RODRIGUES, F.C.M. ; FIGLIOLIA, M.B. (Ed): Sementes florestais tropicais. Brasília: ABRATES, 1993. p.83-136.

BRASIL. Ministério da Agricultura e Reforma Agrária. Regras para análise de sementes. Brasília: SNDA/DNDV/ CLAV, 1992. $365 \mathrm{p}$.

CARNEIRO, N.B.; FERRAZ, I.D.K.; VARELA, V.P. Efeito da temperatura sobre a germinação de sementes de Triplaris surinamensis Cham e Dipteryx alata Vog. In: JORNADA DE INICIAÇÃO CIENTÍFICA DO INPA, 6., 1997, Manaus. Anais... Manaus: Instituto Nacional de Pesquisas da Amazônia, 1997. v.1, p. 219-222.

CARVAlho, N.M.; NAKAGAWA, J. Semente: ciência, tecnologia e produção. 4 ed. Jaboticabal: FUNEP, 2000. 588p.

DUCKE, A. Notas sobre a flora neotrópica II: As leguminosas da Amazônia brasileira. In: IAN. Boletim técnico, 18. 2.ed. Belém: IAN, 1949. 248p.

FERRAZ, I.D.K.; VARELA, V.P. Temperatura ótima para a germinação de sementes de trinta espécies florestais da Amazônia. In: HIGUCHI, N.; SANTOS, J.; SAMPAIO, P.T.B.; MARENCO, R.A.; FERRAZ, J.; SALES, P.C.; SAITO, M.; MATSUMOTO, S. Projeto Jacaranda. Fase II: Pesquisas Florestais na Amazônia Central. Manaus: CPST/INPA, 2003. p.117-127.

FIGLIOLIA, M.B.; OLIVEIRA, E.C.; PINÃ-RODRIGUES, F.C.M. Análise de semente. In: AGUIAR, J.B.; PINÃ-RODRIGUES, F.C.M.; FIGLIOLIA, M.B. (Ed.) Sementes florestais tropicais. Brasília: ABRATES, 1993. p.173-174.

GENTIL, P.F.; TORRES, S.B. Umedecimento do substrato e germinação de sementes de maxixe (Cucumis anguria L.). Revista Brasileira de Sementes, Brasília, v.23, n.2, p.113-116, 2001.

MAGUIRE, J.O. Speed of germination and in selection and evaluation for seedling emergence and vigor. Crop Science, Madison, v. 2, n.2, p.176-177, 1962.

MARCOS FILHO, J. Germinação de sementes. In: SEMANA DE ATUALIZAÇÃO EM SEMENTES, 1. , 1986, Campinas. Anais... Campinas: Fundação Cargill, 1986. p.11-39.

MAYER, A.M.; POLJAKOFF-MAYBER, A. The germination of seeds. Oxford: Pergamon Press, 1989. 270p.

MESQUITA, L.G. Aspectos tecnológicos e morfológicos da germinação de sementes de angelim pedra (Dinizia excelsa Ducke). 44f. (Monografia) - Instituto de Tecnologia da Amazônia, Manaus, 2004.
MIRANDA, P.R.M.; FERRAZ, I.D.K. Efeito da temperatura na germinação de sementes e morfologia da plântula de Maquira sclerophylla (Ducke) C. C. Berg. Revista Brasileira de Botânica, São Paulo, v.22, n.2, p.303-307, 1999. Suplemento.

NOVEMBRE, A.D.L.C.; MARCOS FILHO, J. Estudo da metodologia para condução do teste de germinação em sementes de algodão deslintadas mecanicamente. Revista Brasileira de Sementes, Brasília, v.21, n.2, p.187-193, 1999.

POPINIGIS, F. Fisiologia da semente. Brasília: AGIPLAN, 1985. 289 p.

PHANEENDRANATH, B.R. Influence of amount of water in the paper towel on standard germination tests. Journal of Seed Technology, Lansing, v.5, n.2, p.82-87, 1980.

RAMOS, A.; BIANCHETTI, A. Influência da temperatura e do substrato na germinação de sementes florestais. In: SIMPÓSIO INTERNACIONAL SOBRE PRODUÇÃO E QUALIDADE DE SEMENTES FLORESTAIS, 1984. Curitiba, Anais... Curitiba: UFPR, 1984. p.252-275.

RIZZINI, C.T. Árvores e madeiras úteis do Brasil. São Paulo: EDUSP, 1971.294p.

RONDON, E.V. Comportamento de essências florestais nativas e exóticas no norte do Mato Grosso. In: CONGRESSO E EXPOSIÇÃO INTERNACIONAL SOBRE FLORESTAS 6., 2000, Porto Seguro: RESUMOS TÉCNICOS DO FOREST 2000. BIOSFERA, 2000. p.68.

RONDON, E.V. Produção de biomassa e crescimento de árvores de Schizolobium amazonicum (Huber) Ducke sob diferentes espaçamentos na região de Mata. Revista Árvore, Viçosa, v.26, n.5, p.573-576, 2002.

TANAKA, M.A.S.; MARIANO, M.I.A.; LEÃO, N.V.M. Influência da quantidade de água, no substrato sobre a germinação de sementes de amendoim. Revista Brasileira de Sementes, Brasília, v.13, n.1, p. 73-76, 1991.

VARELA, V.P.; FERRAZ, I.D.K.; CARNEIRO, N. B. Efeito da temperatura na germinação de sementes de sumaúma (Ceiba pentandra L. Gaertn - Bombacaceae). Revista Brasileira de Sementes, Brasília, v. 21, n.2, p.170-174, 1999.

VICENTE, M.; NORONHA, A.; SILBERSCHMIDT, K. Substrate moisture levels for germination testing of some agricultural seeds. Anais da Academia Brasileira de Ciências, Rio de Janeiro, v.41, n.4, p.633-639, 1969. 